\title{
Tiling the Torus and Other Space Forms*
}

\author{
Marjorie Senechal
}

Department of Mathematics, Smith College, Northampton, MA 01063, USA

\begin{abstract}
We consider graphs on two-dimensional space forms which are quotient graphs $\Gamma / F$, where $\Gamma$ is an infinite, 3-connected, face, vertex, or edge transitive planar graph and $F$ is a subgroup of $\operatorname{Aut}(\Gamma)$, all of whose elements act freely on $\Gamma$. The enumeration of quotient graphs with transitivity properties reduces to computing the normalizers in $\operatorname{Aut}(\Gamma)$ of the subgroups $F$. Results include: all isogonal toriodal polyhedra belong to the two families found by Grünbaum and Shephard; there are no transitive graphs on the Möbius band; there is a graph on the Klein bottle whose automorphism group acts transitively on its faces, edges, and vertices.
\end{abstract}

\section{Introduction}

The cylinder, the torus, the Möbius band, and the Klein bottle are the four two-dimensional Euclidean space forms, quotient manifolds of the Euclidean plane $E^{2}$. These manifolds are obtained by identifying points which are equivalent under the action of a discrete subgroup $F$ of $E(2)$, all of whose nontrivial elements act freely on $E^{2}$ (for any $g \in F$ and any $x \in E^{2}, g x=x$ implies $g$ is the identity). The four types of subgroups defining the space forms are, respectively: generated by a single translation (type $l 1$ ); generated by two linearly independent translations (type $p 1$ ); generated by a glide reflection (type $\mathrm{lg}$ ); and generated by a translation together with a glide reflection in a linearly independent direction (type pg) (see [22]). In this paper we present a unified approach to the study of graphs with transitivity properties embedded in these quotient manifolds and give a classification of the graphs whose map automorphism groups act transitively on their faces, edges, or vertices.

\footnotetext{
* This paper is an expanded version of a lecture presented to the Conference on Combinatorial Geometry, Oberwolfach, Germany, September 1984.
} 
The graphs $\gamma$ we consider are quotient graphs $\Gamma / F$, where $\Gamma$ is an infinite 3 -connected face, vertex, or edge transitive graph embedded in $E^{2}$ and $F$ is subgroup of $\operatorname{Aut}(\Gamma)$ whose elements act freely, as described above. Sirch planar graphs have long been known and have been studied in detail [4][6], [11], [15], [18]. As Figs. 1-3 suggest, they can always be embedded in the plane so that $\operatorname{Aut}(\Gamma) \subset E(2)$. Since $\operatorname{Aut}(\Gamma)$ is infinite, discrete, and has compact fundamental region, it is by definition one of the 17 two-dimensional crystallographic groups.

We will show that the problem of determining the transitivity properties of $\gamma$ reduces to the computation of subgroups of these groups [14], [19], [20]. These computations produce some interesting and diverse results, including the following: the symmetry groups of discrete cylindrical patterns, the so-called "rod groups," are quotient groups of type $N / F$ (Section 5.2); all isogonal toroidal polyhedra belong to the two infinite families recently described by Grünbaum and Shephard (Section 5.4); there are no transitive graphs $\Gamma / F$ on the Möbius band (Proposition 4.1); there is a graph on the Klein bottle whose automorphism group acts transitively on its faces, vertices, and edges (Example 3 of Section 4).

In Section 2 we briefly review some of the properties of infinite planar graphs with transitivity properties and their analogues on the quotient manifolds. In Section 3 we discuss map automorphisms and establish some of their properties. In Section 4 we discuss the details of the enumeration of the transitive graphs $\gamma$; the results are presented in Table 2. Finally, in Section 5 we discuss some related problems.

Throughout this paper $\Gamma, \gamma$, and $F$ will always denote graphs and subgroups of the type described above.

\section{The Laves, Kepler, and Heesch Graphs}

Consider a graph embedded in a surface with a 2 -cell embedding. Such a graph is said to be:

(i) a Laves graph if each face is a $k$-cycle, with vertices of valences $\left[v_{1}, \ldots, v_{k}\right]$

(ii) a Kepler graph if each vertex is $j$-valent, and the $j$-faces meeting there are $\left(f_{1}, \ldots, f_{j}\right)$-cycles;

(iii) a Heesch graph if the two vertices incident with each edge are $v_{1}$-and $v_{2}$-valent and the two adjacent faces are $f_{1}$ - and $f_{2}$-cycles; the symbol for such a graph is $\left\{v_{1}, v_{2} ; f_{1}, f_{2}\right\}$.

An infinite 3-connected face, vertex, or edge transitive planar graph $\Gamma$ is clearly a Laves, Kepler, or Heesch graph, respectively. Euler's formula for finite planar graphs, $f-e+v=1$, can be extended to these infinite graphs; it implies that:

If $\Gamma$ is a Laves graph, then the integers $v_{i}$ satisfy the equation

$$
1 / v_{1}+\cdots+1 / v_{k}=k / 2-1
$$




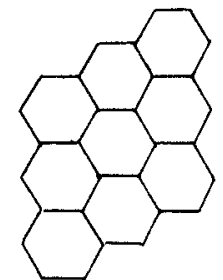

(a) $\left[6^{3}\right]$

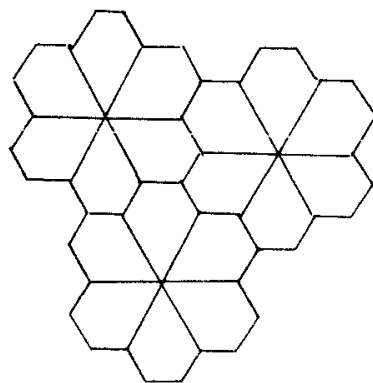

(d) $\left[3^{4} .6\right]$

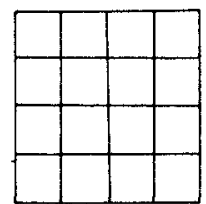

(b) $\left[4^{4}\right]$

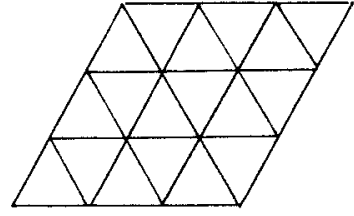

(c) $\left[3^{6}\right]$

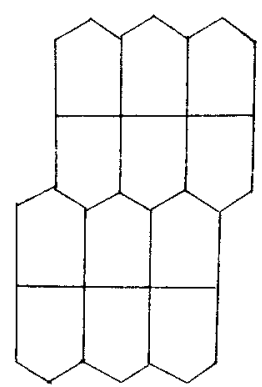

(e) $\left[3^{3} \cdot 4^{2}\right]$

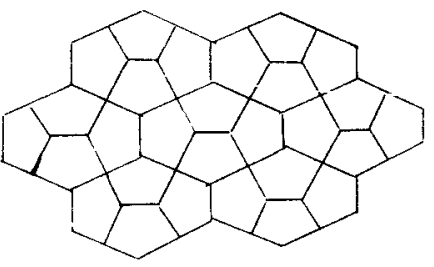

(f) $\left[3^{2} \cdot 4.3 .4\right]$

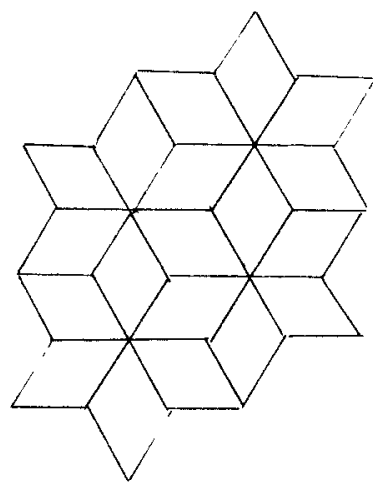

(h) [3.6.3.6]

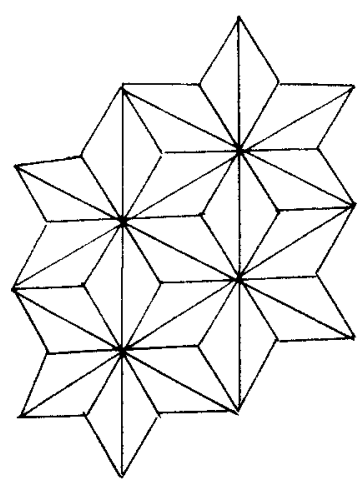

(i) $\left[3.12^{2}\right]$

(g) [3.4.6.4]

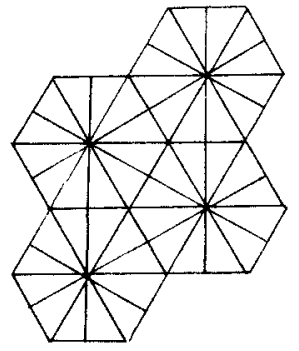

(j) $[4.6 .12]$

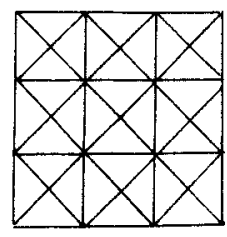

(k) $\left[4.8^{2}\right]$

Fig. 1. The 11 Laves graphs for the plane, characterized by the cyclic ordering $\left[v_{1}, v_{2}, \ldots, v_{k}\right]$ of the valences of the vertices of each face. 


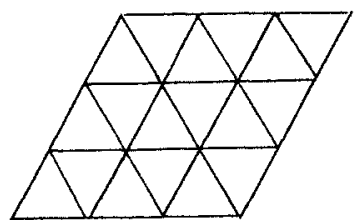

(a) $\left(6^{3}\right)$

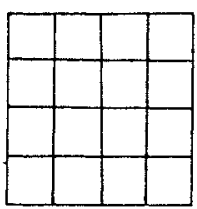

(b) $\left(4^{4}\right)$

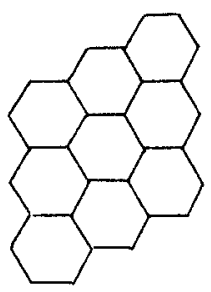

(c) $\left(3^{6}\right)$

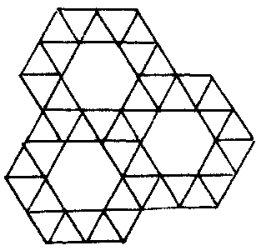

(d) $\left(3^{4} .6\right)$

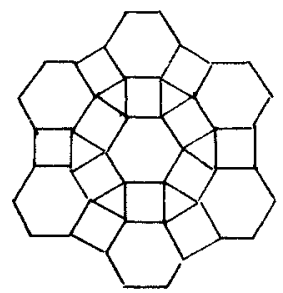

(g) $(3.4 .6 .4)$

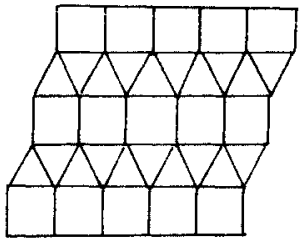

(e) $\left(3^{3} .4^{2}\right)$

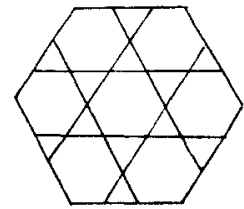

(h) (3.6.3.6)

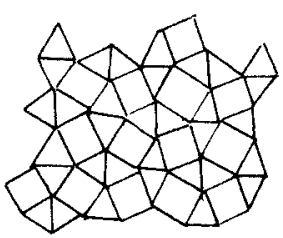

(f) $\left(3^{2} .4 .3 .4\right)$

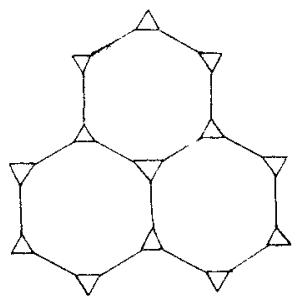

(i) $\left(3.12^{2}\right)$

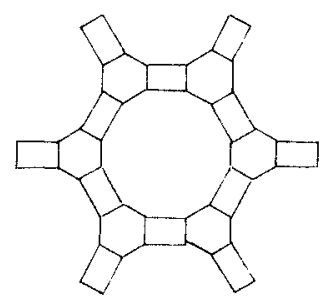

(j) (4.6.12)

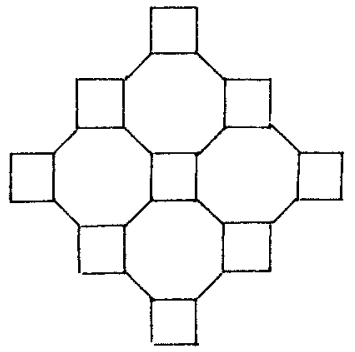

(k) $\left(4.8^{2}\right)$

Fig. 2. The 11 Kepler graphs for the plane, characterized by the cyclic orderings $\left(f_{1}, f_{2}, \ldots, f_{j}\right)$ of the $f$-gons which meet at each vertex. 


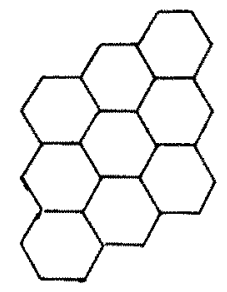

(a) $\{3,3 ; 6,6\}$

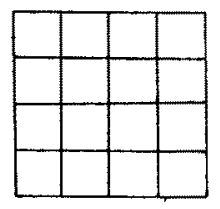

(b) $\{4,4 ; 4,4\}$

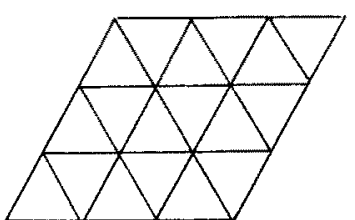

(c) $\{6,6 ; 3,3\}$

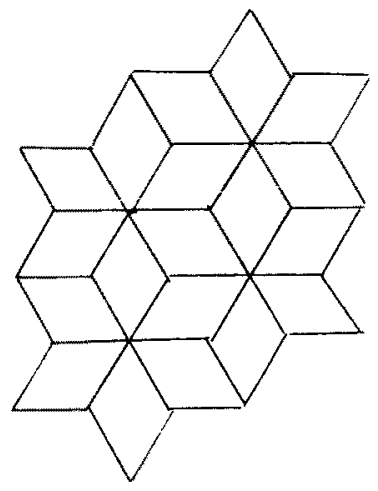

(d) $\{3,6 ; 4,4\}$

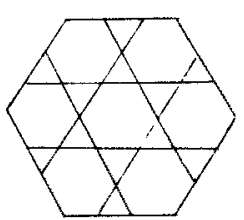

(e) $\{4,4 ; 3,6\}$

Fig. 3. The five Heesch graphs for the plane, characterized by the symbol $\left\{v_{1}, v_{2} ; f_{1}, f_{2}\right\}$ which indicates that the two vertices of each edge are $v_{1}$ - and $v_{2}$-valent, and the edge is shared by an $f_{1}$-gon and an $f_{2}$-gon.

If $\Gamma$ is a Kepler graph then the integers $f_{i}$ satisfy the equation

$$
1 / f_{1}+\cdots+1 / f_{j}=j / 2-1 \text {, }
$$

and if $\Gamma$ is a Heesch graph then

$$
1 / v_{1}+1 / v_{2}+1 / f_{1}+1 / f_{2}=1
$$

The solutions of these equations which correspond to graphs are shown in Figs. 1-3.

In fact, equations (2.1), (2.2), and (2.3) also characterize the Laves, Kepler, and Heesch graphs $\gamma$ on the cylinder, torus, Möbius band, and Klein bottle. For the torus and Klein bottle these equations follow when the requirements of uniform face, vertex, or edge cycles are combined with Euler's condition $f-e+$ $v=0$. Only those solutions can be realized in $E^{2} / F$ which can also be realized in $E^{2}$, since any graph embedded in the quotient manifold can be unfolded to a planar graph with the same properties. The argument is a little more complicated for graphs on the cylinder or Möbius band, since these graphs are infinite. However, if we assume that the graphs are normal (see [7]) then the techniques used to obtain equations $(2.1),(2.2)$, and (2.3) for planar graphs can be applied and we obtain the same results. 


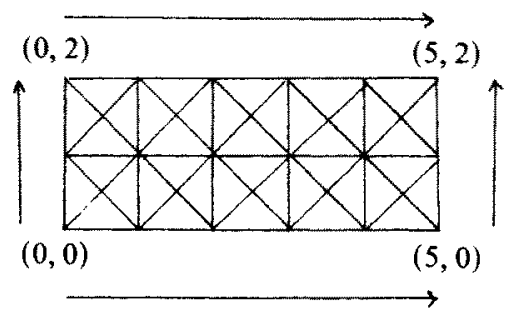

Fig. 4. A toroidal quotient graph of type $\left[4.8^{2}\right]$. The graph is face and vertex transitive, but not edge transitive.

It is a remarkable fact that, in the plane, these local uniformity conditions ensure transitivity; this does not happen on the sphere, nor does it occur in $E^{3}$. It need not happen on the space forms either (Fig. 4).

\section{Map Automorphisms}

A map automorphism is one which extends to a homeomorphism of the embedded surface; it maps face boundaries to face boundaries. Every automorphism of $\Gamma$ is a map automorphism, but the map automorphisms of a quotient graph may be a proper subgroup of its automorphism group. We denote the group of map automorphisms of a quotient graph $\gamma$ on $E^{2} / F$ by $\operatorname{Aut}_{M}(\gamma)$. If $\gamma$ is a Laves, Kepler, or Heesch graph, then any element of $\operatorname{Aut}_{M}(\gamma)$ can be interpreted as an automorphism of the unfolded (covering) graph $\Gamma$ in $E^{2}$ which maps $F$-orbits (of faces, vertices, or edges) onto $F$-orbits. Thus every map automorphism of $\gamma$ is induced by an automorphism of $\Gamma$. Conversely, assume that $F \subset \operatorname{Aut}(\Gamma)$. If $g \in \operatorname{Aut}(\Gamma)$ maps $F$-orbits to $F$-orbits, then it induces a map automorphism of $\Gamma / F$, since it maps points identified under $F$ onto one another and the entire graph onto itself. We now show that this implies that $\operatorname{Aut}_{M}(\gamma)$ is isomorphic to $N_{\text {Aut }(\Gamma)}(F) / F$,

Proposition 3.1. Let $G$ be a discrete subgroup of $E(2), H$ a subgroup of $G$, and $g \in E(2)$. Then $g$ maps H-orbits (of points of $E^{2}$ ) to orbits if and only if $\mathrm{gHg}^{-1}=\mathrm{H}$.

Proof. Let $x \in E^{2}$, and denote its orbit under $H$ by $\{H x\}$. Suppose that for some $g \in E(2),\{g H x\}$ is also an $H$-orbit. Since this orbit contains $g x$ it is the orbit $\{H g x\}$ and hence $\{g H x\}=\{H g x\}$. Thus for $x \in E^{2}$ and $f \in F$, there is an $f_{x} \in F$ such that $f g x=g f_{x} x$. Then $f_{x}^{-1} g^{-1} f g x=x$, so $f_{x}^{-1} g^{-1} f g$ is in the $G$-stabilizer of $x$. Since $G$ is discrete, we are guaranteed the existence of an $x$ with trivial stabilizer and hence $f_{x}^{-1} g^{-1} f g$ is the identity. Thus $f_{x}=g^{-1} f g$ for all $x$. The converse is immediate.

Corollary 1. Let $\Gamma$ be a Laves, Kepler, or Heesch graph, $F$ a subgroup of Aut $(\Gamma)$, and $g \in \operatorname{Aut}(\Gamma)$. If $g$ maps F-orbits (of faces, vertices, or edges of $\Gamma$ ) to F-orbits, then $g \in N=N_{\text {Aut }(\Gamma)} F$. 
Proof. This follows from Proposition 3.1 and the fact that $\operatorname{Aut}(\Gamma) \subset E(2)$.

Corollary 2. $\operatorname{Aut}_{M}(\gamma)$ is isomorphic to $N / F$.

Corollary 3. $\operatorname{Aut}_{M}(\gamma)$ acts transitively on $\gamma$ if and only if $N$ acts transitively on $\Gamma$.

Proof. If a group acts transitively on a set then any of its quotient groups acts transitively on the quotient set, and conversely.

Example. The toroidal Laves graph of Fig. 4 is not transitive. The covering graph is $\left[4.8^{2}\right]$, shown in Fig. 2(k); Aut $(\Gamma)$ is $p 4 m$ and $F$ is the subgroup generated by the two translation $(5,0)$ and $(0,2)$. The normalizer $N$ of $F$ in $\operatorname{Aut}(\Gamma)$ is a pmm subgroup of $p 4 \mathrm{~m}$; it is easy to see that $N$ does not act transitively on the faces of $\Gamma$, since an orbit of $\mathrm{pmm}$ can have faces of only two different orientations.

The following fact is very useful:

Proposition 3.2. If $H \subset \operatorname{Aut}(\Gamma)$ acts transitively on the faces, vertices, or edges of $\Gamma$ then $[\operatorname{Aut}(\Gamma): H]=\left[S: S^{\prime}\right]$, where $S$ is the stabilizer subgroup in $\operatorname{Aut}(\Gamma)$ of a face, vertex, or edge $\mu$ or $\Gamma$, and $S^{\prime}=H \cap S$.

Proof. For each $\mu_{i} \in \Gamma$ there is a $g_{i} \in \operatorname{Aut}(\Gamma)$ such that $g_{i} \mu=\mu_{i}$. Thus $\{g \in$ $\left.\operatorname{Aut}(\Gamma) \mid g \mu=\mu_{i}\right\}$ is the coset $g_{i} S$, and $\operatorname{Aut}(\Gamma)$ is the union of all such cosets. Since $H$ acts transitively it must contain $|S|$ elements from each coset.

\section{Computation}

Our task is to determine which of the automorphism groups of the graphs $\Gamma$ of Figs. 1-3 have $l 1, p 1$, lg, or $p g$ subgroups $F$ and in each case whether the normalizer $N$ of $F$ in Aut( $\Gamma)$ acts transitively on $\Gamma$.

Let $\mu$ be a face, vertex, or edge of a Laves, Keepler, or Heesch graph $\Gamma$. Since no nontrivial element of $F$ fixes any point, there is a one-one correspondence between the elements of the $F$-orbit $\left\{F_{\mu}\right\}$ and the elements of the group $F$. Let $S$ be the stabilizer subgroup in $N$ of $\mu$. Then the stabilizer of $\{F \mu\}$ is $R=F \rtimes S$, the semidirect product of $F$ by $S . R \subset N$ since $F \subset N$ and $S \subset N$. The stabilizers of different $F$-orbits are conjugate. Since there is a one-one correspondence between the faces, vertices, or edges of $\gamma$ and the cosets of $R$ in $N, \operatorname{Aut}_{M}(\gamma)$ is the image of the permutation representation of $N$ defined by the subgroup $R$. $N$ and $F$, together with $\Gamma$ and $R$, completely describe $\gamma$, since the numbers and arrangement of faces, edges, and vertices are specified by $\Gamma, F$, and the permutation representation.

We first briefly review some facts about crystallographic groups.

As we noted in the introduction, an $n$-dimensional crystallographic group $G$ is a discrete subgroup of $E(n)$ with compact fundamental region. We will use here, however, an equivalent characterization: (1) $G$ is an extension of a free 
abelian group $T=Z^{n}$ of rank $n$ (which we identify with a discrete group of translations of $E^{n}$ ) by a finite group $P$ isomorphic to a subgroup of $O(n)$; (2) the mapping $\varphi: P \rightarrow \operatorname{Aut}(T)$ which defines the extension is an injection.

If $H$ is any subgroup of $G$, then it is an extension of $T_{H}=T \cap H$ by a subgroup $P_{H}$ of $P$, and $[G: H]=\left[P: P_{H}\right]\left[T: T_{H}\right] . H$ is crystallographic if the induced action $\varphi_{H}: P_{H} \rightarrow \operatorname{Aut}\left(T_{H}\right)$ is an injection. If $H$ is an invariant subgroup of $G$ then it must be crystallographic; for an elementary proof see [11].

Three of the four discrete fixed-point-free subgroups of $E(2)$ are crystallographic: $l 1, p 1$, and $p g . l g$ is not crystallographic because $P_{H}$ acts trivially on $T_{H}$. We conclude that:

Proposition 4.1. There are no transitive Laves, Kepler, or Heesch graphs on a Möbius band.

Proof. By Proposition 3.2, if $N$ acts transitively on $\Gamma$ then $\left[T: T_{N}\right]$ is finite. Hence $N$ contains translations in two linearly independent directions from which it follows that $N$ is a crystallographic group [19]. Therefore $N$ cannot have an invariant $l g$ subgroup.

In fact, the normalizer of an $\lg$ subgroup is a band (or frieze) group; thus transitive graphs on a Mobius band are inherently one dimensional.

If $T=T_{H}$ then $H$ is said to be a translation-equivalent subgroup of $G$

Proposition 4.2. If $F$ is an $l 1$ or $p 1$ subgroup of $G$ then its normalizer is a translation-equivalent subgroup of $G$.

Proof. Since $F \subset T$ and since $T$ is abelian, we have $N_{G}(F) \supset T$.

Therefore, in order to identify $\operatorname{Aut}_{M}(\gamma)$ when $F$ is of type $l 1$ or $p 1$, we need only look for the maximal translation-equivalent transitive subroup of $\operatorname{Aut}(\Gamma)$ in which $F$ is normal.

According to Grünbaum and Shephard, two transitive infinite plane patterns are said to belong to the same (henomeric) plane pattern type if they have the same symmetry group, the same stabilizer subgroup, and the same motif-transitive subgroups [8]. Since these are precisely the groups that enter into our enumeration of quotient graphs with transitivity properties, we may consider, instead of the covering graphs of Figs. 1-3, the 11 pattern types to which they correspond (Table 1). The types of motif-transitive subgroups of the symmetry groups of the plane pattern types are listed in [8]; this, together with Proposition 3.2 and its obvious corollary $|\mathrm{S}| /\left|S^{\prime}\right|=\left[P: P_{H}\right]\left[T: T_{H}\right]$, makes it possible to identify the possible normalizers $N$. After determining the lattices of these subgroups [14], we check their pg subgroups to see which are in fact the correct normalizers [21]. Then for each pattern type we list (Table 2) those admissible pairs $(N, F)$ for which $N$ is a translation-equivalent subgroup of Aut $(\Gamma)$. (Thus, by Proposition 4.2, the list is complete for $l 1$ and $p 1$ subgroups $F$, but not for $p g$ subgroups.) In each case we also give $R$, and $[N: R]$ when this index is finite. The structure of $N / F$ 
Table 1. The pattern types of planar Laves, Kepler, and Heesch graphs.*

\begin{tabular}{|c|c|c|c|c|c|c|}
\hline \multirow{2}{*}{$\begin{array}{l}\text { Plane } \\
\text { pattern } \\
\text { type }\end{array}$} & \multirow{2}{*}{$\begin{array}{l}\text { Stabilizer } \\
\text { of motif }\end{array}$} & \multirow{2}{*}{$\begin{array}{l}\text { Symmetry } \\
\text { group }\end{array}$} & \multirow{2}{*}{$\begin{array}{l}\text { Laves, Kepler, or } \\
\text { Heesch graph } \Gamma\end{array}$} & \multicolumn{3}{|c|}{$\begin{array}{l}\text { Existence of } \\
(N, F)\end{array}$} \\
\hline & & & & $l 1$ & $p 1$ & $p g$ \\
\hline 19 & $D 1$ & $\mathrm{cmm}$ & {$\left[3^{3} \cdot 4^{2}\right],\left(3^{3} \cdot 4^{2}\right)$} & $\sqrt{ }$ & $\sqrt{ }$ & $\sqrt{ }$ \\
\hline 35 & $D 1$ & $p 4 g$ & {$\left[3^{2} \cdot 4.3 .4\right],\left(3^{2} \cdot 4.3 .4\right)$} & $\sqrt{ }$ & $\sqrt{ }$ & $\sqrt{ }$ \\
\hline 38 & $D 1$ & $p 4 m$ & {$\left[4.8^{2}\right],\left(4.8^{2}\right)$} & $\sqrt{ }$ & $\sqrt{ }^{\prime}$ & $\sqrt{ }$ \\
\hline 40 & $D 2$ & $p 4 m$ & $\{4,4 ; 4,4\}$ & $\sqrt{ }$ & $\sqrt{ }$ & $\sqrt{ }$ \\
\hline 41 & $D 4$ & $p 4 m$ & {$\left[4^{4}\right],\left(4^{4}\right)$} & $\sqrt{ }$ & $\sqrt{ }$ & $\sqrt{ }$ \\
\hline 42 & $C 1$ & $p 6$ & {$\left[3^{4} \cdot 6\right],\left(3^{4} \cdot 6\right)$} & $\ldots$ & $\sqrt{ }$ & - \\
\hline 46 & $C 1$ & $p 6 m$ & {$[4.6 .12],(4.6 .12)$} & - & $\sqrt{ }$ & - \\
\hline $\begin{array}{c}48 \\
(A \text { or } B)\end{array}$ & $D 1$ & $p 6 m$ & $\begin{array}{l}{[3.4 .6 .4],(3.4 .6 .4),} \\
\{3,6 ; 4,4\},\{4,4 ; 3,6\}, \\
{\left[3.12^{2}\right],\left(3.12^{2}\right)}\end{array}$ & - & $\sqrt{ }$ & - \\
\hline 49 & $D 2$ & $p 6 m$ & $\begin{array}{l}{[3.6 .3 .6],(3.6 .3 .6),} \\
\{3,3 ; 6,6\},\{6,6 ; 3,3\}\end{array}$ & - & $\sqrt{ }$ & 一 \\
\hline 50 & D3 & $p 6 m$ & {$\left[3^{6}\right],\left(3^{6}\right)$} & $\sqrt{ }$ & $\sqrt{ }$ & $\sqrt{ }$ \\
\hline 51 & D6 & $p 6 m$ & {$\left[6^{3}\right],\left(6^{3}\right)$} & $\sqrt{ }$ & $\sqrt{ }$ & $\sqrt{ }$ \\
\hline
\end{tabular}

* The infinite planar Laves, Kepler, and Heesch graphs are realizations of 11 different plane pattern types. In the first column we give the pattern type number (Grünbaum and Shephard [8]), and in the second and third columns their stabilizer subgroups and symmetry grups. The corresponding Laves, Kepler, and Heesch graphs are listed in column four. In column five we indicate whether the symmetry group has a motif transitive subgroup $H$ containing an invariant subgroup $F$ of type $l 1$, pi or pg. (The admissible pairs $(N, F)$ are described in detail in Table 2.)

is given in [21]. In part (a) of Table $2, F$ is type $l 1$, in part (b), $F$ is type $p 1$, and in part (c), $F$ is type $p g$. The case $F=l g$ is not considered since these subgroups are never invariant in $\operatorname{Aut}(\Gamma)$.

In Table 2 the invariant subgroups are identified by their generators. For this purpose we need to know $T_{H}, P_{H}$ and how they are put together. First recall [20] that an element of a crystallographic group $G$ can be written in the form $(t+\tau, p)$ where $t \in Z^{n}, \tau \in R^{n}$, and $p \in p$. Multiplication in $G$ follows the rule

$$
\left(t_{1}+\tau_{1}, p_{1}\right)\left(t_{2}+\tau_{2} p_{2}\right)=\left(t_{1}+\tau_{1}+\bar{p}_{1}\left(t_{2}+\tau_{2}\right), p_{1} p_{2}\right)
$$

$\left(\bar{P}=\varphi P\right.$, where $\varphi$ is the injection $\varphi: P \rightarrow \operatorname{Aut}(T)$.) The elements $\tau_{i}$ must satisfy the Frobenius congruence

$$
\tau_{1}+\bar{p}_{1} \tau_{2} \equiv \tau_{3} \quad(\bmod T)
$$

where $p_{1} p_{2}=p_{3}$. We write $t *_{1,2}=\tau_{1}+\bar{p}_{1} \tau_{2}-\tau_{3}$. (If $G$ is a semidirect product, then the $\tau$ 's can always be chosen so that $t *$ is equal to 0 ). Now let $H$ be a subgroup of $G$. Then every element of $H$ has the form $\left(t_{H}+t+\tau, p_{H}\right)$ where $t_{H} \in T_{H}$, $t \in T, p_{H} \in P_{H}$, and $\tau$ is the "fractional" translation associated with $p_{H}$ as an element of $P$. The translations $t$ satisfy a congruence analogous to (4.1):

$$
t_{1}+\bar{p}_{1} t_{2} \equiv \overline{p_{1} p_{2}} t_{3}+t *_{1,2} \quad\left(\bmod T_{H}\right)
$$

for every $p_{1}, p_{2} \in P_{H}$. 
Table 2. Transitive graphs on space forms. (In this table, vector components refer to the "natural" basis for the translation subgroup of Aut( $\Gamma$ ) (see [19].)

\begin{tabular}{clll}
\hline $\begin{array}{c}\text { Plane } \\
\text { pattern } \\
\text { type }\end{array}$ & \multicolumn{1}{c}{$F$} & & \\
\hline 19 & $(l 1)$ & $N$ & $R$ \\
\hline & $\langle(a, b)\rangle$ & $p 2$ & $F$ \\
& $\langle(a, a)\rangle$ & $c \mathrm{~cm}$ & $F \times D 1$ \\
35 & $\langle(a,-a)\rangle$ & $c \mathrm{~m}$ & $F \times D 1$ \\
38 & $\langle(a, 0)\rangle$ & $p g g$ & $F$ \\
40 & $\langle(a, a)\rangle$ & $\mathrm{cmm}$ & $F$ \\
41 & $\langle(a, a)\rangle$ & $\mathrm{cmm}$ & $F$ \\
& $\langle(a, b)\rangle$ & $p 2$ & $F \times C 2$ \\
& $\langle(a, 0)\rangle$ & $p m m$ & $F \times D 2$ \\
50 & $\langle(a, a)\rangle$ & $\mathrm{cmm}$ & $F \times D 2$ \\
& $\langle(a, b)\rangle$ & $p 2$ & $F \times C 2$ \\
& $\langle(a, 0)\rangle$ & $\mathrm{cmm}$ & $F \times D 2$ \\
51 & $\langle(a,-a)\rangle$ & $\mathrm{cmm}$ & $F \times D 2$ \\
& $\langle(a, b)\rangle$ & $p 2$ & $F \times C 2$ \\
& $\langle(a, 0)\rangle$ & $\mathrm{cmm}$ & $F \times D 2$ \\
& $\langle(a,-a)\rangle$ & $\mathrm{cmm}$ & $F \times D 2$ \\
\hline
\end{tabular}

(b) Transitive graphs on the torust

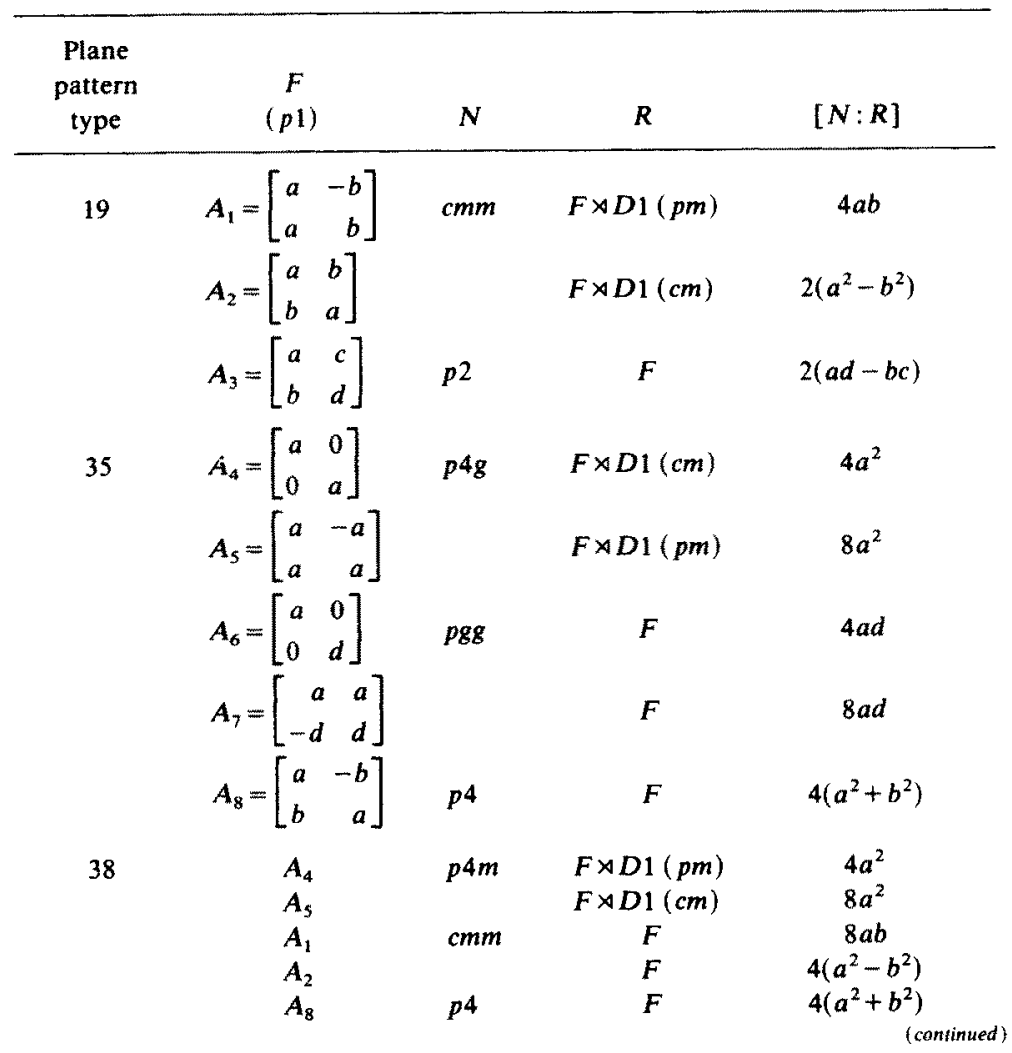


Table 2 (continued)

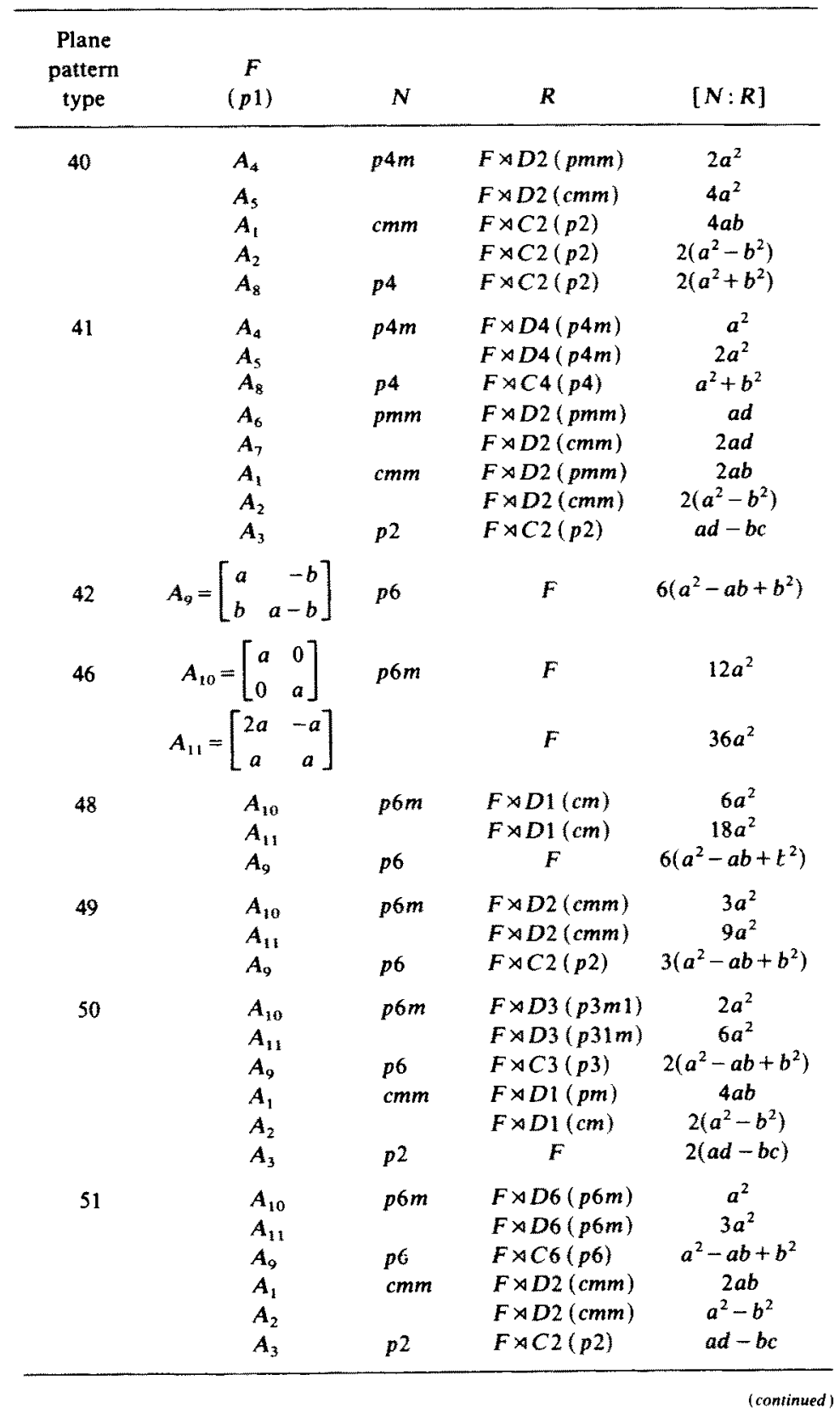


Table 2 (continued)

(c) Transitive graphs on the Klein bottle (with translation-equivalent $N$ ) $\neq$

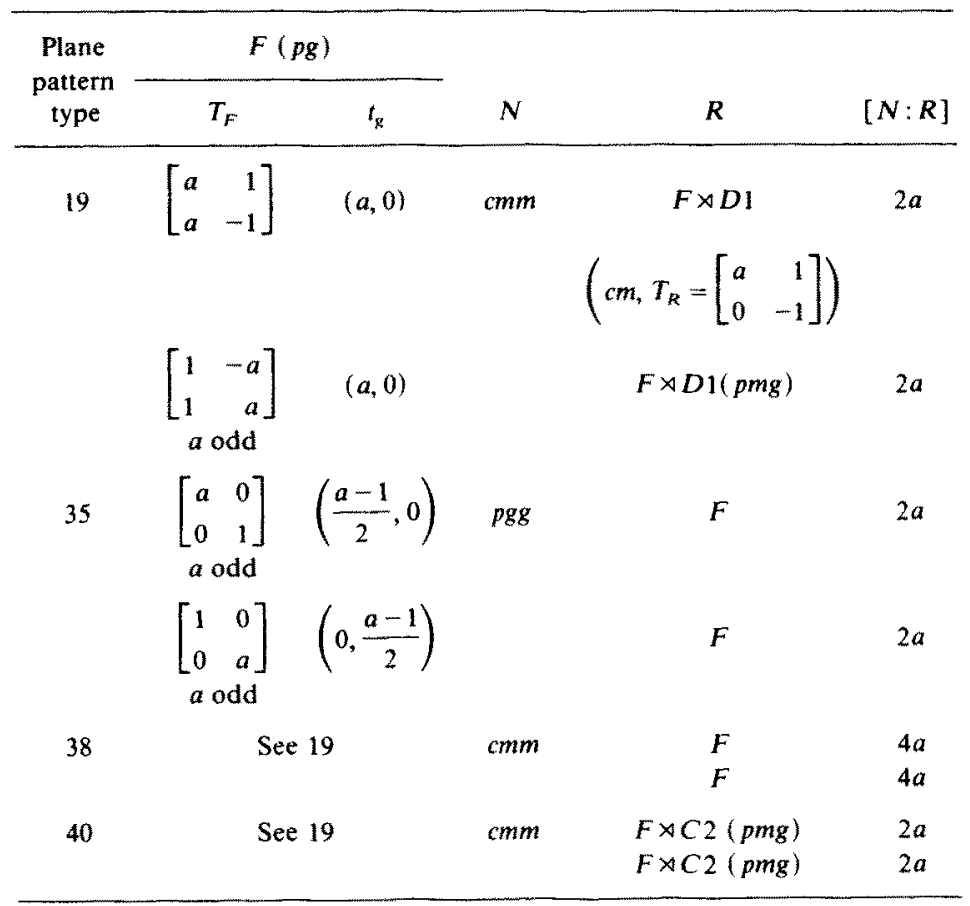

* In columns 2 and 3 , for each pattern type (column 1) we list the admissible pairs $(F, N)$ where $N$, a motif-transitive subgroup of the symmetry group $G$ of the plane pattern (see Table 1), is the normalizer of $F$. $F$ is an $l 1$ subgroup, generated by a single translation.

$\dagger$ The first four columns are as in (a), except that here $F$ is a $p 1$ subgroup of $G$. Since $R$ is of finite index in $N$ we list $[N: R]$ in column 5 ; this index is the number of motifs (faces, vertices, or edges) of $\gamma$. Since $N$ is a translationequivalent subgroup of $G$ it is completely identified by its isomorphism type. $¥$ We list only those graphs for which $N$ is a translation-equivalent subgroup of $G$. The column headings are the same as in (b). The $p g$ subgroups $F$ are completely characterized by their translation sublattices $T_{F}$ and the solution $t_{\mathrm{g}}$ of the subgroup congruence (see text).

Each solution set $\left\{t_{i}\right\}$ determines a distinct subgroup of $G$. To identify $H$, then, we need to know $P_{H}$, a basis for $T_{H}$ and the solution set $\left\{t_{i}\right\}$ for the generators of $P_{H}$.

When $n=2, T=Z^{2}$ and $P$ is one of the cyclic or dihedral groups $C 1, C 2$, $C 3, C 4, C 6, D 1, D 2, D 3, D 4$, or $D 6$. Therefore, a subgroup is identified by the vector or pairs of vectors which generate $T_{H}$ and by the solution vectors $t_{s}$ and/or $t_{m}$, where $s$ generates the cyclic subgroup of $P_{H}$ and, if $P_{H}$ is dihedral, $m$ is a representative of the coset of $\langle s\rangle$. (A table of $t_{s}$ and $t_{m}$ for all the two-dimensional groups is given in [9].) 
Our results are shown in Table 2 . We conclude this section with examples which illustrate the method used to compile it and how the entries can be interpreted.

Example 1. Vertex-transitive graphs on the cylinder. Six of the 11 vertextransitive graphs $\Gamma$ admit pairs $(N, F)$ where $F$ is type $l 1$. Let us consider graphs of type $\left(3^{6}\right)$. Their quotient graphs are six-regular triangulations of the cylinder; such graphs are of importance in the study of biological and crystalline structures in which subunits are closely packed in rod-like configurations [2], [16]. In Table 1 we find that $\Gamma$ is plane pattern type 50 and its automorphism group is $p 6 \mathrm{~m}$. Let $F$ be an $l 1$ subgroup generated by the translation vector $(a, b), a, b \in Z$. (The components are relative to unit translation vectors which form an angle of $120^{\circ}$.) $F$ is not an invariant subgroup of $\operatorname{Aut}(\Gamma)$ itself since the rotations of $p 6 \mathrm{~m}$ map $(a, b)$ to vectors in other orientations. However, Aut $(\Gamma)$ has, up to conjugacy, three proper translation equivalent subgroups which act transitively on $\Gamma$ and do have invariant $l 1$ subgroups. One is a $p 2$ subgroup, in which any $l 1$ subgroup is invariant; the others are $\mathrm{cm}$ and $\mathrm{cmm}$ subgroups. If $b \neq a$ (or 0$)$ and $b \neq-a$, then the $p 2$ subgroup is the normalizer $N$ of $F=\langle(a, b)\rangle$. Relative to $p 2$, the stabilizer of a vertex is $C 2$, so $R=F \times C 2$. The image $N / F=\operatorname{Aut}_{M}(\gamma)$ is isomorphic to $(Z x C k) \times C 2$, where $k$ is the greatest common divisor of $a$ and $b$. The symmetries of this graph are: rotation of order $k$ about the axis of the cylinder, translation or screw rotation along the axis (which has direction $(a-2 b, 2 a-b)$ ), and two-fold rotation about each vertex (Fig. 5(a)).

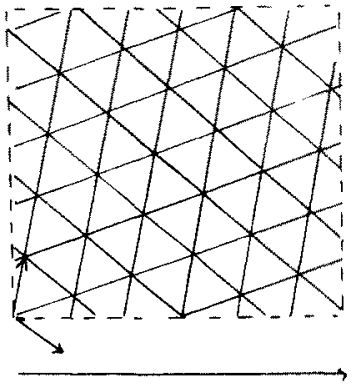

(a)

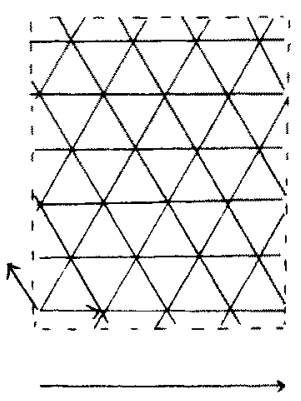

(b)

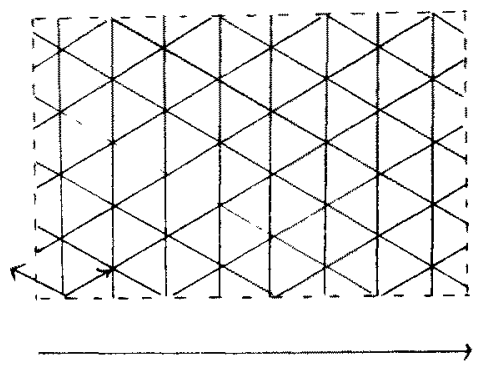

(c)

Fig. 5. Infinite cylindrical quotient graphs of type $\left(6^{3}\right) .($ a) $F$ is generated by the translation $(6,4)$; its normalizer is the translation-equivalent $p 2$ subgroup of Aut $(\Gamma)(p 6 m)$. The axis of the cylinder is a twofold rotation axis. The other automorphisms of this graph are twofold rotation about each vertex, and translation or screw rotation parallel to the cylindrical axis. (b) $F$ is generated by the translation $(4,0)$. The normalizer $N$ is the translation-equivalent $\mathrm{cmm}$ subgroup of Aut $(\Gamma)$. The graph has vertical and horizontal reflection symmetries as well as fourfold rotation about the cylindrical axis and twofold rotation about each vertex. (c) $F$ is generated by $(4,-4) . N$ and the automorphisms are the same as in (b). 
Otherwise, if $b=a$ (or 0$)$, or $b=-a$, then $F=\langle(a, a)\rangle$ and $F=\langle(a,-a)\rangle$ are invariant in the $\mathrm{cm}$ and $\mathrm{cmm}$ subgroups of Aut $(\Gamma)$; in both cases the normalizer of $F$ is the $\mathrm{cmm}$ subgroup. The stabilizer subgroup $S$ is $D 2, R=F \times D 2$, and the image $N / F$ is $(Z x C a) \rtimes D 2$. These cylindrical graphs have vertical and horizontal reflection planes as well as rotations or order $a$ about the cylindrical axis and order 2 about each vertex (Fig. 5(b) and (c)).

Example 2. Face transitive graphs on the torus. By definition every twodimensional crystallographic group has an invariant subgroup of type $p 1$; indeed, it has one or more infinite families of subgroups of this type. Let us consider face-transitive toroidal graphs of type [3 $\left.3^{4} .6\right]$, which is plane pattern type 42 . Since $S$ is trivial, $\operatorname{Aut}(\Gamma)$, which is $p 6$, has no proper transitive subgroups (Proposition 3.2). Therefore, in order for $N$ to be transitive $F$ must be invariant under $p 6$. This means that $F$ must define a lattice which is invariant under six-fold rotations. Such a subgroup can be generated by any vector $(a, b)$ together with its image under $120^{\circ}$ rotation, $(-a, a-b)$. In Fig. 6 we see the lattice generated by $(3,2)$ and $(-2,1)$. Since $S=\{1\}, R=F$. The index of $F$ in $\operatorname{Aut}(\Gamma)$ is $6\left(a^{2}-a b+\right.$ $b^{2}$ ) which, since $R=F$, is also the number of faces of $\gamma$. Since each face has five edges, the number of edges of $\gamma$ is $15\left(a^{2}-a b+b^{2}\right)$, and from $f-e+V=0$ we conclude that $v=9\left(a^{2}-a b+b^{2}\right)$. To describe $F$ succinctly, we can write its generators as columns of an integral $2 \times 2$ matrix $A$. It is well known [10] that all other pairs of generators of $F$ are represented by the columns of the set of matrices $\{A X\}, X \subset G L(2, Z)$. Since $\gamma$ is obtained from $\Gamma$ simply by identifying points in $F$-orbits, it is independent of the choice of generators. Thus every matrix $A X$ defines the same graph.

This is the general procedure for obtaining all Laves, Kepler, and Heesch toroidal graphs. To each group $N$ there is associated a matrix $\boldsymbol{A}$ whose determinant prescribes the possible shapes of $F$ and the number of faces, edges, and vertices of $\gamma$. These matrices are fully described in [19].

Example 3. Edge-transitive graphs on the Klein bottle. Of the five families of edge-transitive graphs shown in Fig. 3 , only $\{4,4 ; 4,4\}$, plane pattern type 40 , has an automorphism group ( $p 4 \mathrm{~m}$ ) with edge-transitive subgroups which contain subgroups of type $\mathrm{pg}$; there are four of them, of types $\mathrm{cmm}, \mathrm{cm}, \mathrm{pgg}$, and $\mathrm{pmm}$. The first and second of these are translation equivalent subgroups, and are the only ones we will consider. The $p g$ subgroups which are invariant in the $\mathrm{cm}$ subgroup are also invariant in the $\mathrm{cmm}$ subgroup (see [21]), so $\mathrm{cmm}$ is the normalizer for all of them. For this pattern the stabilizer subgroup of $\mathrm{cmm}$ is $C 2$, so $R=F \rtimes C 2$, a pmg subgroup of index $2 a$ in $N$ (Table 2(c)). Thus for each of the two families of graphs with $N=c m m, \gamma$ has $2 a$ edges, $a$ faces, and $a$ vertices.

It is interesting to note (see Table 2(a) and (b)) that the $\mathrm{cmm}$ subgroup acts transitively not only on the edges, but also on the faces and vertices of $\gamma$. Accordingly, the corresponding automorphism group $\operatorname{Aut}_{M}(\gamma)$ acts transitively 


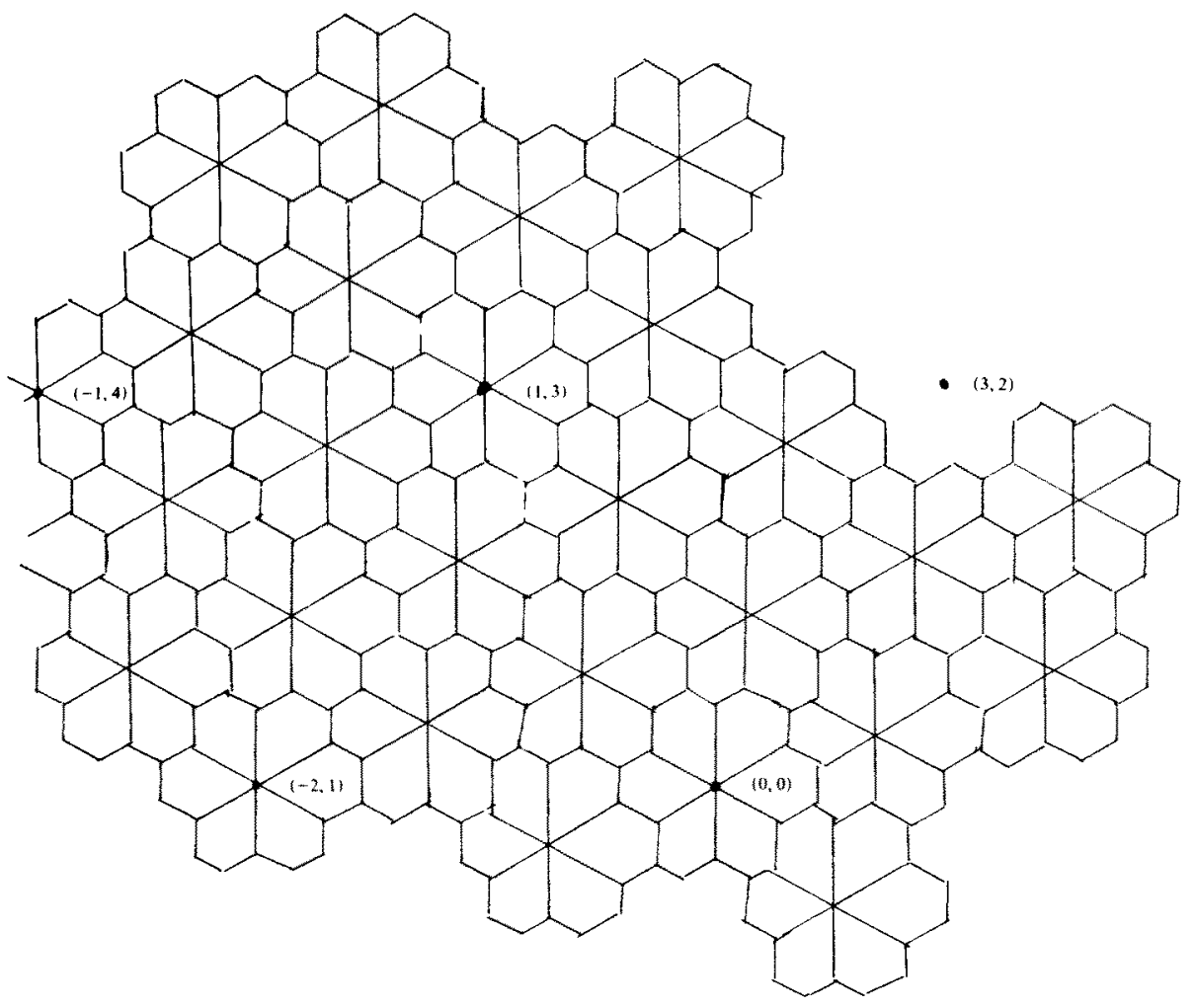

Fig. 6. Identification of the points of the lattice $\left[\begin{array}{rr}3 & -2 \\ 2 & 1\end{array}\right]$ gives us a toroidal quotient graph of type $\left[3^{4} .6\right]$. The graph can be represented by any parallelogram which contains points of this lattice only at its vertices. This graph has 42 faces, 105 edges, and 63 vertices.

on the faces, vertices, and edges of $\gamma$ but not, however, on its flags (a flag is a triple $(v, e, f)$ with $v \subset e \subset f)$; flag transitivity would require $N=\operatorname{Aut}(\Gamma)$.

\section{Discussion}

We briefly discuss several interesting topics related to this work. These include the relation between $\operatorname{Aut}_{M}(\gamma)$ and Aut $(\gamma)$, the crystallographic rod groups, perfect colorings of transitive graphs $\gamma$, the realizability of the graphs $\gamma$ in $E^{3}$, and generalizations to higher dimensions.

\section{1. $\operatorname{Aut}_{M}(\gamma) \operatorname{Aut}(\gamma)$}

This work was originally undertaken to investigate the conjecture, inspired by [3], that the only automorphisms of embedded quotient graphs which are not 
map automorphisms are those which arise from identifications that occur in "small" graphs. Hutchinson [13] has shown that if the face and edge widths $K$ and $r$ of an embedded toroidal graph are sufficiently large then all its automorphisms are map automorphisms. For face, vertex, and edge regular graphs on the torus this is always the case if $K>6$ and $r>12$; in most cases smaller values suffice. $K$ and $r$ are independent of the choice of generators of $F$, and thus Table $2(b)$ is a complete enumeration of "large" toroidal graphs with transitivity properties. What can be said about graphs on the cylinder and the Klein bottle? It is reasonable to conjecture that an analogous result can be found in the first case. However, Table 2(c) and auxiliary calculations show that the width of transitive graphs on the Klein bottle are small. This suggests that these graphs may have automorphisms which are not map automorphisms. Thus the "nice" algebraic behavior of the orientable space forms strongly contrasts with the behavior of the nonorientable ones.

\subsection{The Discrete Rod Graphs and Cylindrical Patterns}

Since the cylinder is a developable surface, realizations of any of the 51 plane pattern types can be rolled into a cylindrical pattern, and the methods of this paper can be used to study these patterns and their symmetries. Indeed, every infinite discrete subgroup of $E(3)$ which leaves a line invariant must be isomorphic to a quotient group $N / F$ since any cylindrical pattern on which is acts transitively can be unfolded to a periodic transitive pattern in the plane, and every transitive cylindrical pattern must be a "quotient pattern." These "one-dimensional" subgroups of $E(3)$ are known as rod groups; they were first investigated by Hermann [12]. They are usually described as extensions of $Z$ by a finite subgroup of $O(3)$; in our formulation they are extension of $Z \times C k$ by a cyclic or dihedral group $P$ (see Section 4). Only special cases of cylindrical patterns have been investigated [2], [16].

\subsection{Perfectly Colored Laves, Kepler, and Heesch Graphs on $E^{2} / F$}

A perfectly colored embedded plane pattern is one in which each motif is assigned a color and each isometry of the pattern effects a permutation of the colors. Perfectly colored patterns are classified in the first instance by their color groups, that is, by the permutation representations of the symmetry groups $G$ which the coloring defines. In a perfectly colored pattern the motifs of a single color form an orbit of a subgroup $H$ of finite index in $G$, and the sets of motifs of the other colors are the images of this orbit under representatives of the left cosets of $H$ in $G$. The colors are permuted by left coset multiplication. Thus the color groups of plane patterns are pairs $(G, H)$. We require that $H$ contain the stabilizer $S$ of a motif [19] to ensure that each motif receives a single color.

All of this applies to planar Laves, Kepler, and Heesch graphs if we regard the faces, edges, or vertices of $\Gamma$ as motifs, and it carries over to quotient graphs 
if instead of isometry groups we consider map automorphism groups. The color group of a colored graph $\gamma$, then, is a pair $(N / F, H / F)$ where $F \subset R \subset H \subset N$. It is a straightforward exercise to find all subgroups $H$ (the construction of lattices of subgroups of crystallographic groups is discussed in [14]).

\subsection{Realizability}

It is an open question which of the cylindrical and toroidal graphs of Table 2(b) can be realized with plane convex faces in $E^{3}$. For example, for toroidal graphs of type $\left(3^{6}\right)$, with $v=a^{2}-a b+b^{2}$, the minimum number of vertices is seven although three is also of this form; according to Brehm [1], a six-regular torus of arbitrary shape must have at least five vertices. Realizability is clearly a function of width; it would be of interest to make this relationship precise.

If, in addition to realizability, we insist on transitivity under some subgroup of $E(3)$, then, in contrast to the cylinder, there are only two (infinite) families of toroidal polyhedra, both vertex transitive. Every finite set of points in $E^{3}$ on which some isometry group acts transitively must lie on the surface of a sphere; thus there are no face or edge transitive polyhedral tori. Vertex transitive tori must be constructed from Kepler graphs which contain no $n$-gons with $n>4$, since only the vertices of triangles and quadrilaterals can be simultaneously coplanar and cospherical; thus the toroidal polyhedra must be derived from the planar graphs $\left(3^{6}\right),\left(3^{3} .4^{2}\right),\left(4^{4}\right)$, and $\left(3^{2} .4 .3 .4\right)$. Furthermore, the vertices must lie on two parallel small circles of the sphere. Families derived from the first two graphs do exist [9]; no vertex transitive graphs of the second two types are possible.

\subsection{Higher-Dimensional Analogues}

There are ten discrete fixed-point-free subgroups in $E(3)$ [22]. If we knew the analogues of the Laves, Kepler, and Heesch graphs in three-dimensional space, it would be possible to determine admissible pairs $(N, F)$ and thus classify transitive tessellations of the three-dimensional space forms. Unfortunately, our ignorance of tessellations of $E(3)$ is almost complete. It should be possible, however, to study the problem for some classes of tessellations that are known; the recent work of Kuhnel and Lassmann [17] provides a starting point.

\section{Acknowledgments}

I am indebted to Joan Hutchinson and Werner Nowacki for many stimulating discussions, and to H. S. M. Coxeter, Branko Grünbaum, Fred Holroyd, and the referees for helpful comments on earlier versions of the manuscript. 


\section{References}

1. U. Brehm, Personal communication.

2. R. O. Erickson, Tubular packing of spheres in biological fine structure, Science 181 (1973), 705-716.

3. E. Gethner and J. P. Hutchinson, Platonic graphs on the torus, unpublished.

4. B. Grünbaum and G. C. Shephard, The eighty-one types of isohedral tilings in the plane, Math. Proc. Cambridge Philos. Soc. 82 (1977), 177-196.

5. B. Grünbaum and G. C. Shephard, The ninety-one types of isogonal tilings in the plane, Trans. Amer. Math. Soc. 242 (1978), 335-553.

6. B. Grünbaum and G. C. Shephard, Isotoxal tilings, Pacific J. Math. 76 (1978), 407-430.

7. B. Grünbaum and G. C. Shephard, The geometry of planar graphs, in Combinatorics (H. N. Y. Temperly, ed.) (Eight British Combinatorial Conference, Swansea 1981), 124-150, Cambridge University Press, Cambridge, 1981.

8. B. Grünbaum and G. C. Shephard, A hierarchy of classification methods for patterns, Z. Krist. 154 (1981), 163-187.

9. B. Grübaum and G. C. Shephard, Polyhedra with transitivity properties, Math. Rep. Canad. Acad. Sci. V1 (1984), 61-66.

10. G. Hardy and E. M. Wright, An Introduction to the Theory of Numbers, 4th ed, 26-29, Oxford, 1960.

11. H. Heesch, Uber topologisch gleichwertige Kristallbindungen, Z. Krist. 84 (1933), 399-407.

12. K. Hermann, Zur systematischen Struktur Theorie. IV. Untergruppen, Z. Krist. 69 (1929), 533-555.

13. J. P. Hutchinson, Automorphism properties of embedded graphs, J. Graph Theory 8 (1984), 35-49.

14. M. V. Jarić and M. Senechal, Space groups and their isotropy subgroups, J, Math. Phys. 25 (1984), 3148-3154.

15. J. Kepler, Harmonices Mundi, 1619.

16. E. Koch and W. Fischer, Types of sphere packings for crystallographic point groups, rod groups, and layer groups, $Z$. Krist. 148 (1978), 107-152.

17. W. Kuhnel and G. Lassmann, The rhombidodecahedral tessellation of 3-space and a particular 15-vertex triangulation of the 3-dimensional torus, Manuscripta Math. 49 (1984), 61-77.

18. F. Laves, Ebenenteilungen and Koordinationszahl, Z. Krist. 78 (1931), 208-241.

19. M. Senechal, Color groups, Discrete Appl. Math. 1 (1979), 51-73.

20. M. Senechal, A simple characterization of the subgroups of space groups, Acta Cryst. Sect. A 36 (1980), 845-850.

21. M. Senechal, Morphisms of crystallographic groups: kernels and images, J. Math. Phys. 26 (1985), 219-228.

22. J. A. Wolf, Spaces of Constant Curvature, 4th ed., Publish or Perish, Berkeley, 1977.

Received March 22, 1985, and in revised form December 3, 1986. 